\title{
Supuestos epistemológicos y ontológicos presentes en la historia de la ecología
}

\author{
Carolina I. García Curilaf ${ }^{12,2}$ \& Guillermo M. Denegri ${ }^{2}$ \\ ${ }^{1}$ FilosofíadelaCiencia, DepartamentodeFilosofía,FacultaddeHumanidades, UniversidadNacionaldeMardelPlata, Buenos \\ Aires,Argentina. ${ }^{2}$ CONICET,LaboratoriodeZoonosisParasitarias, DepartamentodeBiología,FacultaddeCienciasExactasy \\ Naturales-UNMdP. Mar del Plata, Buenos Aires, Argentina.
}

\begin{abstract}
RESUMEN. El propósito de este trabajo es mostrar los supuestos epistemológicos (empirista, racionalista y racioempirista) y ontológicos (individualista, holista y sistemista) presentes en la historia de la ecología, sobre la base de tres trabajos: Clements (1916), Gleason (1926) y Lindeman (1942). En este artículo analizamos el surgimiento de una posición moderada en la historia de la ecología. Defendemos la tesis de que a partir del trabajo publicado por Lindeman en 1942, comenzó a surgir en ecología un marco conceptual que toma implícitamente un enfoque ontológico sistemista y epistemológico racioempirista, y que se encuadra en los principios filosóficos desarrollados por Bunge. También mostramos las desventajas de implementar la epistemología racionalista y la epistemología empirista en la investigación ecológica, y las ventajas de utilizar la epistemología racioempirista. En la actualidad, los programas de investigación en ecología siguen alguno de estos tres principios ontológicos y epistemológicos.
\end{abstract}

[Palabras clave: empirismo, racionalismo, racioempirismo, holismo, individualismo, sistemismo]

\begin{abstract}
Aвstract. Epistemological and ontological assumptions present in the history of ecology. The purpose of this paper is to show the epistemological assumptions (empiricists, rationalists and ratioempirist) and ontological (individualistic, holistic and systemist) present in the history of ecology, analyzing three jobs (Clements 1916; Gleason 1926; Lindeman 1942). This article analyzes the emergence in the history of the ecology of a moderate position. We defend the thesis that from the work developed by Lindeman in 1942 began to emerge in ecology a conceptual framework that implicitly takes a systemist ontological and racioempirista epistemological approach, and fits into the philosophical principles developed by Bunge. We also show the disadvantages of implementing the rationalist and empiricist epistemology in ecological research, and the advantages of using the ratioempiricism epistemology. Currently, research programs in ecology follow one of three ontological and epistemological principles.
\end{abstract}

[Keywords: empiricism, rationalism, ratio-empiricism, holism, individualism, systemism]

\section{INTRODUCCIÓN}

Desde sus comienzos, la ecología tuvo desacuerdos en torno a cuál debía ser su objeto de estudio y cómo debía ser estudiado. Este debate perdura en la actualidad y constituye el centro que define y regula a la ecología como ciencia. Dependiendo del enfoque epistemológico y ontológico que se tome para estudiar la ecología de individuos, de poblaciones, de comunidades y de ecosistemas, se obtendrán determinados resultados y diferentes concepciones de lo que es la naturaleza y de cómo debe ser estudiada. Este artículo analiza los problemas ontológicos y epistemológicos que han dominado la historia de la ecología desde sus comienzos. En particular, se trata el problema ontológico de comunidades y de ecosistemas. Es decir, si las comunidades y los ecosistemas son meros agregados de Editor asociado: Jobbágy Esteban

carolinagarcia49@gmail.com organismos, como sostiene el individualismo, o si bien las comunidades y ecosistemas realmente existen, como sostiene el holismo. Por otra parte, también trata el problema epistemológico de si la ecología debe ser una ciencia puramente descriptiva de búsqueda de patrones o una ciencia especulativa dedicada a realizar conceptualizaciones con una prueba fenomenalista de sus términos teóricos, teorías e hipótesis. Este artículo analiza el surgimiento en la historia de la ecología de una posición moderada que tiene en cuenta a ambos enfoques ontológicos y epistemológicos, y, con mayor precisión, defiende la tesis de que a partir del trabajo desarrollado por Lindeman en 1942 comienza a surgir un marco conceptual que implícitamente toma un enfoque ontológico sistemista y un enfoque epistemológico racioempirista o empiriorracionalista. Éstos pertenecen a

Recibido: 2 de marzo de 2016

Aceptado: 23 de agosto de 2016 
los principios filosóficos desarrollados por Mario Bunge. El presente artículo promueve el enfoque ontológico sistémico y epistemológico racioempirista de Bunge debido a que propone la implementación de hipótesis mecanicistas con alto contenido teórico explicativo de los procesos ecológicos, sumando a esto su contrastación empírica. Además, la ontología sistémica en ecología sostiene que las comunidades y los ecosistemas son sistemas interactuantes, que deben ser estudiados tanto en su micro-nivel como en su macro-nivel. Así, la ecología asegura su estatus científico y su crecimiento cognitivo al proponer una síntesis que contenga las ventajas de ambos enfoques epistemológicos y ontológicos. En la actualidad, estos tres enfoques epistemológicos y ontológicos siguen presentes en la investigación ecológica, como detallaremos en la sección Conclusiones.

\section{EL ENFOQUE ONTOLÓGICO HOLISTA Y EPISTEMOLÓGICO RACIONALISTA}

Si bien Haeckel creó el término ecología en 1886, la disciplina comenzó a constituirse como una ciencia autónoma a partir de los aportes que botánicos, zoólogos y limnólogos realizaron de manera separada a finales del siglo XIX y principios del XX. En 1953, Eugene Odum realizó una síntesis en la que aplicó a diferentes sistemas ecológicos los mismos principios que provenían de disciplinas variadas. A comienzos del siglo XX, los botánicos se vieron interesados por tratar de explicar el desarrollo de las comunidades de plantas partiendo de un enfoque ontológico holista y epistemológico racionalista. En 1899, Henry Cowles introdujo el término sucesión ecológica, con el que explicó los cambios en la vegetación de un lago en respuesta a las tensiones físicas. Para Cowles (1899), la sucesión ecológica sucedía a través de secuencias de desarrollo ordenado de la vegetación. Según sus propios términos, la comunidad de plantas era dinámica, fortuita y siempre cambiante. Para desarrollar el término sucesión ecológica, este autor tuvo en cuenta las relaciones entre los factores bióticos y los abióticos. Este término fue retomado en los desarrollos teóricos de Clements, Tansley, Lindeman y Odum, y también forma parte de la explicación actual sobre el desarrollo de una comunidad de plantas (Begon et al. 2008).

En 1916, retomando el término sucesión ecológica introducido por Cowles y debido a la necesidad de explicar el desarrollo de la vegetación, Frederic Clements escribió el libro Plant Succession. An Analysis of the Development of Vegetation, una obra clave en la historia de la ecología ya que Clements es el padre de la escuela que toma un enfoque ontológico holista y epistemológico racionalista (aún hoy en vigencia) (Marone 2007; Begon et al. 2008). Sobre la base de la razón y la invención de términos teóricos, Clements creó una teoría sobre el desarrollo de la vegetación, y por medio de la misma él explicó los patrones que observaba. Clements detectó que una región se caracterizaba por poseer una vegetación particular seleccionada por el clima, que determina la forma de vida de las plantas. La existencia de diferentes comunidades fue pensada por Clements como una teoría del cambio que llamó desarrollo. El término técnico para este proceso fue sucesión ecológica. Para Clements, las comunidades se desarrollan hasta llegar a una fase final denominada clímax climático. Clements realizó una analogía entre el clímax climático de una comunidad de plantas y un organismo individual. Él llamó clímax climático a un organismo complejo (súper-organismo) para distinguir esto de un organismo individual. El ciclo de vida de la comunidad de plantas resulta análogo al del organismo: nace, crece, se desarrolla y senesce. Clements llevó la analogía al extremo, sosteniendo que las comunidades de plantas son organismos complejos. Para Clements (1916), la sucesión ecológica es un proceso lineal hasta llegar a un predecible clímax climático. De todas maneras, cuando la comunidad de plantas llega al clímax climático puede sufrir desequilibrios debido, sobre todo, a factores externos a la comunidad. Esto no impide que la formación vuelva a reiniciar las etapas sucesionales hasta llegar de nuevo a un estado de equilibrio. Clements (1916) puso el foco de esta teoría en la biota y en las interacciones bióticas, y a través de ellas explicó la dinámica de las comunidades vegetales. En su teoría, los factores ambientales fueron considerados sólo como un elemento secundario. Así podemos observar cómo en esta teoría se encontraba presente la epistemología racionalista, caracterizada por la utilización de términos teóricos muy abstractos que pertenecen al terreno de la especulación, ya que sólo se justifican en teorías y no en contrastaciones empíricas. Además, la contrastación de las hipótesis termina siendo fenomenalista (Marone 2007). A través de la creación de términos teóricos como sucesión ecológica, clímax climático, organismo complejo, estabilidad, equilibrio, 
comunidad, entre otros, Clements explicó el desarrollo de las comunidades vegetales. Debido a que estos términos teóricos tienen poco sustento empírico, terminó cayendo en la construcción de teorías antirrepresentacionales y contrafáctica de alto contenido teórico y con poco o nulo contenido empírico, lo que resulta una contradicción para una ciencia fáctica como la ecología.

Por otra parte, Clements asumió un enfoque ontológico holista organicista. El organicismo sostiene que los organismos vivos son complejas estructuras jerárquicas y que las partes son integradas funcionalmente y coordinadas por el todo. Las propiedades y el comportamiento de las partes pueden ser explicados sólo en términos de su funcionamiento en el todo; contribuyen al funcionamiento adecuado, a la supervivencia y a la reproducción de ese todo (Clements 1916; Mayr 2004). Para la ontología holista no se puede reducir el todo a sus partes. Por el contrario, los holistas son antirreduccionistas y se los llama autonomistas porque defienden la autonomía de la biología con respecto a la física y a la química (Ayala 1968; Campbell 1974; Mayr 2004). El holismo ontológico enfatiza la existencia de propiedades emergentes que no pueden predecirse desde el más completo conocimiento de sus partes componentes. La ontología holista radical que sostenía Clements consideraba que se debían estudiar sólo las entidades de alto nivel de organización, las comunidades, y que dicho conocimiento se puede utilizar para explicar las entidades de bajo nivel de organización (poblaciones e individuos). Para el holista radical, las comunidades y los ecosistemas son entidades discretas, integradas, análogas a un organismo con la capacidad de autorregularse. Clements describió a la comunidad de plantas como un organismo que, como tal, tiene cierta fisiología integrada que viene dentro de su existencia y le permite crecer, madurar y entrar en un estado estable que llama clímax climático.

Resulta evidente que la ecología comenzó con una visión holista de la naturaleza, que conlleva una epistemología racionalista. El tipo de conocimiento se aleja de las ciencias fácticas y se acerca a la pseudociencia (Bunge 2000). El holismo ontológico en ecología falla por ser antianalítico y por sostener la tesis errónea de que todo está conectado con todo, inclinándose a filtrar el irracionalismo en la ciencia (Mahner and Bunge 1997).

\section{LA ONTOLOGÍA INDIVIDUALISTA Y LA EPISTEMOLOGÍA EMPIRISTA}

En 1926, Henry Gleason, gran crítico de la teoría de Clements, elaboró un concepto sobre el desarrollo de la comunidad de plantas, y lo publicóensuartículo TheIndividualistic Concept of the Plant Association. Según este autor, la comunidad de plantas es un ensamblaje de individuos, y el clímax climático clementsiano no es predecible ya que cada especie posee un rango único de tolerancia al ambiente. Además, Gleason demostró que la dispersión de las semillas es puramente azarosa. Por lo tanto, una comunidad es el resultado de los cambios de la migración y de la selección por el ambiente, $y$, en cierto modo, resulta menos predecible. Basándose en la observación y no en la especulación metafísica, Gleason presentó una concepción individual de la comunidad vegetal (Gleason 1926). Para él, los ecólogos de su tiempo se basaban en especulaciones metafísicas en lugar de realizar observaciones y experimentos. En este punto residían, según Gleason (1926), todos los desacuerdos y discusiones de la ecología. Este autor críticaba a los ecólogos que, como Clements, veían a la comunidad de plantas como un organismo complejo. Ellos fallaban porque ni siquiera podían definir con precisión cuáles eran sus límites, dónde terminaba una comunidad y comenzaba otra. Por eso resulta necesario volver al concepto individualista de la evolución de las comunidades vegetales (Gleason 1926). En contraposición a la teoría de Clements, Gleason consideraba que la comunidad de plantas no existía y que es, en última instancia, el resultado de condiciones azarosas y cambiantes como la migración y los factores ambientales. Contrariamente a la teoría de Clements, Gleason sostenía que la comunidad de plantas era una asociación heterogénea debido a fluctuaciones climáticas y de migración que, además, cambiaba de manera continua. Por lo tanto, Gleason proponía una concepción individualista de cómo se dispersan y se desarrollan las comunidades de plantas, basándose en hechos empíricos (según sus propias palabras) y no en especulaciones metafísicas (como hicieron Clements y otros ecólogos). Gleason concluyó que la asociación entre plantas comenzaba desde un individuo casual. Esta concepción individualista consideraba que la comunidad no existía y que era más bien un ensamblaje de individuos resultado de cambios en la migración y la selección natural, y que es menos predecible. Como observamos, 
el reduccionismos radical en ecología fue introducido por Gleason en 1926, quien formuló conceptos individualistas de las comunidades de plantas, argumentando que éstas comenzaron desde especies individuales que se adaptaron a las condiciones del ambiente. Más tarde, estas afirmaciones se conocieron como la hipótesis individualista.

El reduccionismo ontológico extremo, denominado atomismo, practicado por Gleason consideraba que las entidades de más bajo nivel eran fundamentales por ser los cimientos del universo, mientras que las entidades de alto nivel eran meros derivados. De esto se deduce que todos los términos, leyes y teorías que se han desarrollado para los altos niveles de organización pueden ser reducidos a conceptos, leyes y teorías que se han desarrollado para los bajos niveles de organización. En consecuencia, la biología se reduce a la física y a la química. El reduccionismo considera que sólo cuando se determina la función de cada una de las partes componentes se puede explicar lo que se observa en los niveles de organización más elevados. El enfoque ontológico individualista lleva implícito un enfoque epistemológico empirista ya que su análisis se enfoca sólo en la descripción y en la búsqueda de los patrones de las poblaciones y de los individuos, y no en la creación de términos teóricos. Si bien la búsqueda de patrones resulta esencial para la ecología, no explica las causas del patrón observado, que debe ser su base, para, de esta forma, determinar por qué surgen ciertas regularidades en el comportamiento de la realidad. Siguiendo los principios de la epistemología empirista, Gleason utilizó el método inductivo, que de casos particulares permite arribar a una conclusión general. En este caso, el patrón al que llegó Gleason fue que la comunidad de plantas no existía y que era, en última instancia, un ensamblaje de individuos resultado del azar y de cambios en los factores ambientales. A su vez, la concepción individualista de la comunidad vegetal podía ser usada como una ley para describir y predecir.

Otras de las características del enfoque epistemológico empirista presente en la teoría de Gleason es la nula formulación de hipótesis (Marone and Bunge 1998). Debido a que este tipo de investigación se justifica en la toma de datos (es decir, en la observación), se evidencia poco contenido teórico. Esto provoca un empobrecimiento de la ecología como ciencia ya que muchos de los procesos ecológicos necesitan ser explicados a través de la formulación de hipótesis, de términos teóricos y de teorías.Por otra parte, el enfoque epistemológico empirista promueve la construcción de teorías antirrepresentacionales dado que no explica las causas de los fenómenos ecológicos. Debido a su superficialidad cognitiva, este tipo de conocimiento puede conducir a los ecólogos a concepciones erróneas de lo que es la naturaleza. Esto lleva a que la ecología sea sólo una ciencia aplicada dedicada a obtener predicciones útiles, resultando en una posición instrumentalista y fenomenalista (Bunge 1985, 2000; Mahner and Bunge 1997; Marone and Bunge 1998; Marone 2007). El producto final de este tipo de investigación son afirmaciones muy dependientes de las condiciones iniciales (Marone 2007). Si las condiciones iniciales cambian, la teoría no puede dar cuenta de esos cambios $y$, en consecuencia, resulta inútil. En esta investigación empírica sólo se describen patrones. Por lo tanto, no se explican los procesos que causan tal patrón. Por lo tanto, esto provoca un empobrecimiento de la ecología como disciplina científica.

\section{LA ONTOLOGÍA SISTEMISTA Y LA EPISTEMOLOGÍA RACIOEMPIRISTA}

La síntesis de todas las teorías desarrolladas por los ecólogos de diferentes disciplinas fue realizada por el limnólogo Raymond Lindeman en 1942, en su artículo The TrophicDynamicAspectEcology.Allí,Lindemanamplió las ideas de sucesión de Cowles, Clements y Tansley, las ideas de dinamismo trófico de Elton y las ideas de la biogeoquímica de Hutchinson. El objetivo de este apartado es mostrar que en la investigación de Lindeman comenzó a surgir en la ecología el enfoque epistemológico racioempirista y ontológico sistémico desarrollado por Mario Bunge (Bunge 1959, 1979, 1985, 1995, 1997, 1999, 2000; Marone 2000; Mahner and Bunge 1997; Marone and Bunge 1998).

Durante años, Lindeman estudió el pequeño lago Cedar Bog para acumular información sobre cómo los distintos componentes bióticos y abióticos estaban integrados al ecosistema. Este estudio le permitió identificar con mayor facilidad las especies, determinando sus hábitos de alimentación. Para poder organizar a las especies en grupos alimentarios tomó como base metodológica la observación y la experimentación. Lindeman fue el primero en implementar de manera explícita el concepto de ecosistema, creado por Tansley en 1935, en 
un esfuerzo cuantitativo para definir, describir y entender el comportamiento dinámico del sistema de ese pequeño lago. A través de la utilización del concepto de ecosistema pudo organizar una enorme cantidad de datos biológicos, como la distribución y abundancia de plantas y animales en el lago dentro de un patrón. Además, Lindeman (1942) creó un esquema bajo observaciones y experimentaciones rigurosas. En ese trabajo enfatizó la interrelación de las partes vivas con las partes no vivas del lago, y concluyó que todas ellas estaban interconectadas por medio de las relaciones tróficas.

A través del concepto de dinamismo trófico, Lindeman introdujo el flujo de energía y los ciclos de la materia. El concepto de ecosistema incluía la idea de que los nutrientes entran y salen del sistema a través de mecanismos de trasporte de la atmósfera, la hidrósfera y la biósfera, y que los organismos vivos (como sub-componentes) están dentro de un sistema tomando, almacenando y liberando nutrientes al medio ambiente. En este camino, las partes vivas están compuestas de las partes no-vivas del sistema, que funciona alternativamente como fuente o suministro de elementos (Lindeman 1942).

Para Lindeman, el ecosistema no sólo es la unión de los animales y de las plantas, sino también entre la biota y el medio ambiente. De un año al otro, Lindeman notó que los grupos dominantes o las especies dentro de un grupo diferían ampliamente. Él explicó que estas variaciones eran causadas por factores externos al lago, como la entrada de agua, la lluvia, la temperatura del invierno, la capa de hielo y el flujo químico. A través de los dinamismos tróficos, Lindeman convirtió a la biomasa de las especies en unidades de energía. La dinámica de los niveles tróficos implementó el concepto de ecosistema, mostrando que los individuos pueden ser estudiados y descriptos en él. Este sistema tiene una estructura compuesta por especies vivas y materiales no-vivos. Los cambios que ocurren en el ecosistema se denominaban sucesión, y se producían de la interacción entre la biota y la materia no-viva con el clima externo, los nutrientes y los disturbios humanos.

Por otra parte, Lindeman clarificó la idea de que los ecosistemas se desarrollan a través de la sucesión ecológica, estando vinculados a la dinámica de los sistemas y el concepto de ciclos de nutrición como ciclos alimentarios. Su programa sostenía que la naturaleza estaba organizada en ecosistemas, que eran objetos reconocibles (como los lagos), que tenían un origen y un desarrollo que conducía a un estado estable o equilibrio dinámico. Estos sistemas poseían una estructura definida por una red de relaciones alimentarias entre las poblaciones de especies. Dichas relaciones podían simplificarse agrupando las poblaciones dentro de cadenas alimentarias. El ecosistema tenía un comportamiento, más allá de la evolución en el tiempo, que implicaba la transformación de la energía recibida del sol u otro sistema, en calor que se almacena y sale del sistema. Entre las especies que conformaban la comunidad existían flujos de energía y ciclos de nutrientes (Lindeman 1942).

Lindeman introdujo muchas de las mayores cuestiones y conceptos de las modernas ecologías energéticas (e.g., la longitud de las cadenas alimentarias, la eficiencia de las transferencias tróficas, el almacenamiento de energía de los diferentes niveles, la tasa de productividad primaria, el problema de la corrección de los valores de energía por perdida debido a la respiración, a la depredación y a la descomposición). En el estudio realizado por Lindeman se puede detectar la implementación de la epistemología racioempirista en la investigación ecológica, ya que la interpretación que realiza de los datos obtenidos en el lago Cedar Bog se efectúa desde una posición moderada tomando elementos del racionalismo y del empirismo. Siguiendo el enfoque epistemológico racioempirista, Lindeman pudo explicar el funcionamiento dinámico del ecosistema del lago a través de la teoría de dinamismo trófico, usando conceptos tales como ecosistemas, sucesión, niveles tróficos, ciclos de energía y ciclos de materia y de la utilización de hipótesis, que puso a prueba bajo la recolección de datos y de experimentos. Otro de los elementos presentes era la implementación de hipótesis mecanicistas (Bunge 1997) para explicar las causas del patrón observado (Mahner and Bunge 1997; Marone and Bunge 1998; Marone 2007). Por otra parte, también implementaba una posición moderada entre el individualismo y el holismo ontológico, denominado sistemismo ontológico (Bunge 1979, 1995; Mahner and Bunge 1997), que admitía la existencia de comunidades y de ecosistemas, pero consideraba necesario analizarlos en términos de sus compontes, ambientes y estructuras (Mahner and Bunge 1997). A través de la toma de datos y de la experimentación realizada en el lago Cedar 
Bog, Lindeman evaluó la hipótesis que propone que las partes vivas y no vivas que conforman el ecosistema del lago se encontraban unidas e interconectadas a través de las relaciones tróficas.

Tal como plantea el enfoque epistemológico racioempirista de Mario Bunge, Lindeman propone una hipótesis mecanicista para explicar el mecanismo que causaba la unión de las partes vivas y las no-vivas en el ecosistema del lago. En este caso, el origen de la interconexión entre ambas partes eran las relaciones tróficas. A través de la experimentación y de la toma de datos, Lindeman confirmó la hipótesis y explicó cómo funcionaba el ecosistema y cómo se relacionaban las distintas partes que lo componían, y pudo también explicar cuál era la función de cada una de las partes integrantes del ecosistema. A través de este estudio se evidenció cómo las relaciones tróficas mantenían unidas a las distintas partes del ecosistema.

La ecología planteada desde un enfoque epistemológico racioempirista y ontológico sistémico resulta más completa y eficaz ya que busca describir, predecir y explicar los fenómenos ecológicos desde un enfoque sistémico que incluya a individuos, poblaciones, comunidades y ecosistemas (Marone and Bunge 2008), haciendo hincapié en la explicación causal que debe ser la base de la ecología. Este enfoque también brinda la ventaja de favorecer la construcción de teorías representacionales de caja traslucida (Bunge 1959, 1985; Marone 2000), es decir, un sistema cuya estructura interna resulta visible. En el caso de la investigación realizada por Lindeman se pudo visualizar cómo las relaciones tróficas interconectan las distintas partes que conforman al ecosistema, pudiendo, a su vez, saber cuál es el rol que cumple cada una las especies en el ecosistema. En contraposición, una teoría de caja negra (Bunge 2000), como la que sostiene el racionalismo o el empirismo, no puede explicar las causas de los fenómenos (Marone 2007). El estudio de Lindeman pudo explicitar lo que de otra forma resultaría inobservable: cómo son las relaciones establecidas entre las distintas partes componentes, el papel que desempeña cada una y el funcionamiento del sistema. De esta forma, al introducir teorías, términos teóricos e hipótesis y someterlos a la prueba empírica, la ecología asegura el crecimiento cognitivo, su eficacia, su precisión y su rigurosidad científica.

\section{CONCLUSIÓN}

A modo de cierre del trabajo recorreremos los principales programas de investigación que sustenta la ecología de comunidades en la actualidad, y vislumbraremos los distintos enfoques epistemológicos y ontológicos que implementan de manera tácita. Debido a la complejidad y a la diversidad del objeto de estudio de la ecología, la investigación ecológica actual es abordada desde los enfoques epistemológicos empiristas y racionalista, y, en menor medida, bajo el enfoque epistemológico racioempirista. Esto se debe a la dificultad para realizar experimentos, ya que no pueden ser repetidos y muchas veces resulta difícil controlar las variables (Diamond 1986; Carpenter et al. 1995). Por su parte, la ecología de comunidades también debe enfrentar el problema de las escalas temporales y espaciales (Cueca 2006; López de Casenave et al. 2007).

Desde la aparición de los experimentos en ecología, los ecólogos han podido comenzar a explicar las causas y los por qué de los patrones observados, así como contrastar de manera más eficaz las hipótesis con alto contenido teórico que propuso el racionalismo. El presente trabajo considera que la realización de experimentos de campo y naturales les permite a los ecólogos manipular las variables del sistema y develar y comprender su rol en la comunidad. Esto es esencial al momento de aplicar los principios de la ecología a políticas de conservación o a la hora de realizar predicciones. La ecología es una ciencia básica y como tal debe tener buenos cimientos cognitivos sobre los cuales construir buena ciencia aplicada.

Hoy en día, una cantidad de programas de investigación en ecología de comunidades toman el enfoque epistemológico propuesto por Bunge. Entre ellos podemos enumerar los estudios de flujo de energía y de materia, los efectos de las especies invasoras, el papel de organismos ingenieros de los ecosistémas, el calentamiento global y los efectos top-down y bottom-up (Alpine and Cloern 1992; Jones et al. 1994; Dyer and Letourneau 1999; Caraco 2007; Anderson and Rosemond 2007). Todos ellos se caracterizan por utilizar contenido teórico explicativo de los procesos ecológicos $\mathrm{y}$, en muchos casos, por la realización de experimentos de campo para poner a prueba las hipótesis planteadas, analizando el todo y la parte. 
Los estudios a largo plazo y a escala de comunidades realizados sobre el cambio climático en la Antártida (McClintock et al. 2008) muestran desde un enfoque epistemológico racioempirista y ontológico sistémico cómo el cambio en el hábitat producido por el aumento de temperatura disminuye la duración y la extensión de capas de hielo marino. Estos cambios drásticos en el hábitat ponen en evidencia cómo se afecta cada uno de los organismos en los distintos niveles de las cadenas tróficas. Gracias a los estudios a largo plazo se pudo comprender el vínculo entre los factores climáticos y la cadena trófica, haciendo posible las predicciones sobre el futuro del ecosistema antártico. Los estudios de largo aliento son fundamentales en ecología de comunidades ya que permiten construir teorías más realistas y más representativas, imprescindibles para explicar y predecir con precisión el impacto de, por ejemplo, el calentamiento global sobre una comunidad. A su vez, los resultados se pueden extrapolar a otras comunidades.

En la actualidad, el enfoque epistemológico empirista se encuentra presente, por ejemplo, en los estudios de la ecología geográfica y macroecología (Underwood 1997; Marone 2007), ya que son disciplinas observacionales que se basan en el análisis estadístico de patrones; estos patrones se generalizan y utilizan para realizar predicciones a partir de observaciones empíricas. A las escalas espaciales y temporales de poco detalle, el investigador tiene dificultades para realizar experimentos y poner a prueba hipótesis mecanicistas que servirían para explicar procesos ecológicos. Los resultados de los estudios macroecológicos se utilizan, por ejemplo, para preservar especies en peligro de extinción y para seleccionar áreas para conservación. Actualmente, los estudios de la ecología aplicada presentan un enfoque epistemológico empirista y ontológico individualista (Peters 1991; Marone 2007).

Por otra parte, en los actuales estudios de ecología de comunidades podemos encontrar el enfoque epistemológico racionalista y ontológico holista en la teoría del nicho y de la competencia, en el principio de equilibrio estable de la comunidad y en la teoría de la sucesión ecológica (que aún hoy incluye un estadío de clímax) (Begon et al. 2008; Marone 2007). Por su alto contenido teórico, la teoría del nicho presenta problemas para su aplicación concreta y se muestra como un término teórico polisémico que cada escuela ecológica utiliza a su manera (Milesi and López de Casenave 2005). En la actualidad, esta teoría está en revisión dado que una cantidad de estudios mostraron dificultades prácticas para determinar el nicho fundamental de una especie (Milesi and López de Casenave 2005).

Agradecimientos. Agradecemos a la Universidad Nacional de Mar del Plata por haber financiado está investigación a través del subsidio EXA EXA764/16.

\section{REFERENCIAS}

Alpine, A., and J. Cloern. 1992. Trophic interactions and direct physical effects control phytoplankton biomass and production in an estuary. The American Society of Limnology and Oceanography 37(5):946-955.

Anderson, C., and A. Rosemon. 2007. Ecosystem engineering by invasive exotic beavers reduces in-stream diversity and enhances ecosystem function in Cape Horn, Chile. Oecologia 154:141-153

Ayala, F. 1968. Biology as an autonomous science. Am. Sci. 56:207-221.

Begon, M., J. L. Harper, and C. R. Towssend. 2008. Ecology: individuals, populations and communities. third edition. Blackwell Science Ltd.

Bunge, M. 1959. Causality. The place of the causal principle in moderns science. Harvard University Press. Cambridge, MA.

Bunge, M. 1979. Ontology II. A world of systems. Dordrecht, Reidel.

Bunge, M. 1985. Teoría y realidad. Ed. Ariel. Barcelona.

Bunge, M. 1995. Sistemas sociales y filosofía. Sudamericana. Buenos Aires, Argentina.

Bunge, M. 1997. Mechanism and explication. Philosophy and social sciences 27:410-465.

Bunge, M. 1999. Las ciencias sociales en discusión. Sudamérica. Buenos Aires, Argentina.

Bunge, M. 2000. La investigación científica. Siglo veintiuno, México.

Cambell, D. 1974. Downward causation in hierarchically organized biological system. Pp 179-1286 In: F. Ayala and Dobzhansky (eds.). Studies in the philosophy of biology. Mac Millan, London.

Caroso, N., J. Cole, and D. Strayer. 2006. Top-down control from the bottom: Regulation of eutrophication in a large river by benthic grazing. The American Society of Limnology and Oceanography 51: 664-670.

Carpenter, S. R., S. W. Chisholm, D. W. Schindler, and R. F. Wright. 1995. Ecosystem experiments, Science 269:324327. 
Clements, F. 1916. Plant Succession: An Analysis of the Development of Vegetation. Published by the Carnegie Institution of Washington, Washington, USA.

Cowles, H. 1899. The Ecology Relations of Vegetation on the Sand Dunes of Lake Michigan. Part I Geographical Relations of the Dunes Floras. Botanical Gazette. 27:95-117.

Cueto, V. R. 2006. Escalas en ecología: su importancia para el estudio de la selección de hábitat en aves. Hornero 21: 001-013

Diamond, J. M. 1986. Overview: Laboratory experiments, field experiments, and natural experiments. Pp. 3-22 in: J. M. Diamond and T. J. Case (eds.). Community ecology. Harper and Row, Nueva York, USA.

Dyer, L., and D. Letourneau. 1999. Trophic cascades in a complex terrestrial community. Proc. Natl. Acad. Sci. 96: 5072-5076.

Gleason, H. A. 1926. The Individualistic Concept of the Plant Association. Bulletin of the Torrey Botanical Club 53: 7-26.

Hillerbrend, H., D. S. Gruner, E. Borere, M. Bracker, E. Cleland, J. Elser, C. Jones, J. Lawton, and M. Shachak. 1994. Organism as ecosystem engineers. Oikos 69:373-386.

Lindeman, R. 1942. The Trophic-Dynamic Aspect of Ecology. Ecology 23:399-417.

López de Casenave J., L. Marone, F. Jaksic, and S. Camus. 2007. Escalas. Pp. 193-213 en: F. Jaksic and L. Marone (eds.). Ecología de Comunidades. Segunda edición ampliada. Ediciones Universidad Católica de Chile, Santiago, Chile.

Mahener, M. and M. Bunge. 1997. Foundations of Biophilosophy. Springer, Berlin.

Marone, L., J. López de Casenave, and R. Gonzales del Solar. Homenaje a Mario Bunge, o por qué las preguntas en Ecología deberían comenzar con por qué. Pp. 153-178 en: G. M. Denegri and G. Martínez (eds.). Tópicos actuales en Filosofía de la ciencia: Homenaje a Mario Bunge en su 80 aniversario. Editorial Martín, Mar del Plata, Argentina.

Marone, L., and M. Bunge. 1998. La explicación en ecología. Boletín Asociación Argentina de Ecología 7:35-37.

Marone, L., J. López de Casenave, and R. Gonzales del Solar. 2007. Qué guía la investigación y profesión ecológica: ¿Los hechos o las ideas? Pp. 53-67 en: A. Arduci, A. Mangione and Lijteroff (eds.). Café ciencia. Editorial de la Universidad Nacional de San Luis, Argentina.

Mayr, D. 2004. Por qué es única la biología. Consideraciones sobre la autonomía de una disciplina científica. Katz. Buenos Aires, Argentina.

Mcclintock, J., D. Hugh, and W. Fraser. 2008. Ecological Responses to Climate Change on the Antarctic Peninsula. American Scientist 96(4):302.

Milesi, F., and J. López de Casenave. 2005. El concepto de nicho en ecología aplicada: del nicho al hecho hay un trecho. Ecología Austral 15:113-148.

Peters, R. H. 1991. A critique for ecology. Cambridge University Press, Cambridge.

Underwood, A. J. 1997. Experiments in ecology: Their logical design and interpretation using analysis of variance. Cambridge University Press, Cambridge. 